\title{
EXTENDING PARTIAL ISOMETRIES
}

\author{
SŁAWOMIR SOLECKI
}

\begin{abstract}
We show that a finite metric space $A$ admits an extension to a finite metric space $B$ so that each partial isometry of $A$ extends to an isometry of $B$. We also prove a more precise result on extending a single partial isometry of a finite metric space. Both these results have consequences for the structure of the isometry groups of the rational Urysohn metric space and the Urysohn metric space.
\end{abstract}

\section{INTRODUCTION}

Let $A$ be a metric space. By a partial isometry of $A$ we mean an isometry between two subsets of $A$. A total isometry, i.e., a partial isometry with domain and range equal to $A$, is called simply an isometry.

In the paper, we answer certain questions which were identified by Henson and by Kechris and Rosendal and which were motivated by, on the one hand, analogies between finite graphs and finite metric spaces and, on the other hand, by problems concerning properties of isometry groups. We prove results of the following three sorts:

1. Given a finite metric space $A$, we extend it to a finite metric space $B$ so that each partial isometry of $A$ extends to an isometry of $B$ (Theorem 2.1 in Section 2).

2. Given a finite metric space $A$ and a partial isometry $p$ on it, we extend $A$ to a finite metric space $B$ so that $p$ extends to an isometry $\tilde{p}$ of $B$ and $A$ and $\tilde{p}^{M}(A)$, for some $M \in \mathbb{N}$, are, in a precise sense, "independent" from each other (Theorem 3.2 in Section 3).

3. We deduce from the results in points 1 and 2 properties of the isometry groups of the rational Urysohn metric space and the Urysohn metric space (Corollaries 4.1, 4.4-4.3 in Section 4).

For more background information the reader should consult the beginning paragraphs of the subsequent sections. Here we recall a couple of definitions

Key words and phrases. Isometries, extension property, Urysohn metric space.

Research supported by NSF grant DMS-0400931. I would like to thank Ward Henson and Alekos Kechris for conversations and emails regarding the paper. Part of this work was done when I visited Caltech in May 2004. I thank the mathematics department there for support.

Accepted in Israel Journal of Mathematics. 
which will be useful throughout the paper. The Urysohn metric space $\mathbb{U}$ is the unique separable metric complete space such that each finite (in fact, separable) metric space embeds isometrically into $\mathbb{U}$ and each isometry between two finite subsets of $\mathbb{U}$ extends to an isometry of $\mathbb{U}[10]$. We call a metric space rational if all the distances between points are rational numbers. Let $\mathbb{U}_{0}$ be the rational Urysohn space, that is, the unique rational metric space which contains an isometric copy of any finite rational metric space (universality) and has the property that any isometry between two of its finite subsets extends to an isometry of the whole space (ultrahomogeneity). For more information on it see [4].

\section{Extending ALL PARTial ISOMETRIES}

In the present section, we will be concerned with the following question:

Given a finite metric space $A$, does there exist a finite metric space $B$ containing $A$ such that each partial isometry of $A$ extends to an isometry of $B$ ?

The problem above came up independently in two different contexts. Pestov proved in [9] that each finite metric space $A$ can be embedded via an approximate isometry into another finite metric space $B$ so that each partial isometry of $A$ approximately extends to an isometry of $B$. (For the precise meaning of "approximate" see [9].) Hrushovski [3] showed that each finite graph $A$ can be isomorphically embedded into another finite graph $B$ so that each partial automorphism of $A$ extends to an automorphism of $B$. Noticing affinities between these two results, Henson asked the question above; positive answer to it would strengthen Pestov's theorem by removing "approximate" and would give the full analogue of Hrushovski's theorem for metric spaces. The second situation in which the same problem surfaced was the study of conjugacy classes of elements of Polish groups carried out by Kechris and Rosendal [5]. In fact, as pointed out by them, an affirmative answer to this question would have consequences for the structure of the conjugacy classes of elements of the isometry groups of the rational Urysohn metric space. These consequences, in turn, have remarkably strong implications for other aspects of the structure of this group (see Corollary 4.3).

In the theorem below, we show that the answer to the question is indeed in the affirmative. (I was informed that A. Vershik announced also proving this theorem.) To prove it we will use the main result of [2]. For model theoretic notation and terminology the reader may consult the first section of [7].

Theorem 2.1. Let $A$ be a finite metric space. There exists a finite metric space $B$ such that $A \subseteq B$ as metric spaces and each partial isometry of $A$ extends to an isometry of $B$. 
Moreover, $B$ can be found so that the distances between points in $B$ belong to the additive semigroup generated by the distances between points of $A$.

Proof. Without loss of generality we assume that $A$ has at least 2 elements. Let $d$ be the metric on $A$. Let $D$ be the set of all positive distances between elements of $A$, that is,

$$
D=\{d(a, b): a, b \in A \text { and } a \neq b\} .
$$

For $r \in D$ let $R_{r}$ be a binary relation. Consider the finite relational language $\mathcal{L}$ consisting of all $R_{r}$ with $r \in D$.

A configuration $\alpha$ is a sequence $r_{0}, r_{1}, \ldots, r_{n}$ of elements of $D$ with the property

$$
\sum_{i=1}^{n} r_{i}<r_{0}
$$

Note that since $D$ is finite and consists of positive numbers, there are only finitely many configurations.

For a configuration $\alpha$ consisting of $r_{0}, r_{1}, \ldots, r_{n} \in D$, let $M_{\alpha}$ be the $\mathcal{L}$ structure with $n+1$ distinct elements $x_{0}, x_{1}, \ldots, x_{n}$ and such that

$$
\begin{aligned}
& M_{\alpha}=\left(R_{r_{0}}\left(x_{0}, x_{n}\right) \text { and } R_{r_{0}}\left(x_{n}, x_{0}\right)\right) \\
& M_{\alpha}=\left(R_{r_{i}}\left(x_{i-1}, x_{i}\right) \text { and } R_{r_{i}}\left(x_{i}, x_{i-1}\right)\right) \text { for } 1 \leq i \leq n
\end{aligned}
$$

and with no other relations $R_{r}$ holding between pairs of elements of $M_{\alpha}$. Let $\mathcal{T}$ consist of all $M_{\alpha}$ for a configuration $\alpha$. Then $\mathcal{T}$ is a finite family of $\mathcal{L}$-structures.

A metric space $X$ with a metric $\sigma$ is made into an $\mathcal{L}$-structure by letting $X \models R_{r}(x, y)$ precisely when $\sigma(x, y)=r$ for $r \in D$ and $x, y \in X$. Note that each isometry of $X$ is an automorphism of $X$ as an $\mathcal{L}$-structure and each partial isometry of $X$ is a partial automorphism. Using the triangle inequality, one easily shows that if a metric space $X$ is considered as an $\mathcal{L}$-structure, then for $M_{\alpha} \in \mathcal{T}$ there is no weak homomorphism $h: M_{\alpha} \rightarrow X$. (Recall that $h$ is a weak homomorphism if for $x, y \in M_{\alpha}, M_{\alpha} \models R_{r}(x, y)$ implies $X \models R_{r}(h(x), h(y))$ for all $r \in D$.) We say that $X$ is $\mathcal{T}$-free.

Notice also that since $D$ includes all positive distances from $A$, any partial automorphism of $A$ as an $\mathcal{L}$-structure is a partial isometry of $A$. Let $\mathbb{U}$ be the Urysohn metric space. We isometrically embed $A$ into $\mathbb{U}$ and extend each partial isometry of $A$ to an isometry of $\mathbb{U}$. Thus, we embedded $A$ as a substructure of a $\mathcal{T}$-free $\mathcal{L}$-structure $\mathbb{U}$ and we extended each partial automorphism of $A$ to an automorphism of $\mathbb{U}$. By [2, Theorem 3.2, p.1994], there exists a finite $\mathcal{T}$-free $\mathcal{L}$ structure $C$ such that $A$ is a substructure of $C$ and each partial automorphism of $A$ extends to an automorphism of $C$. If $p$ is a partial automorphism of $A$, let $\tilde{p}$ be its extension to an automorphism of $C$. We assume that the partial automorphism with empty domain is extended to the identity function on $C$. 
We say that a sequence $c_{0}, \ldots, c_{n} \in C$ is a chain between $c_{0}$ and $c_{n}$ if for each $1 \leq i \leq n$ there exists $r \in D$ with

$$
C \models\left(R_{r}\left(c_{i-1}, c_{i}\right) \text { and } R_{r}\left(c_{i}, c_{i-1}\right)\right) .
$$

Notice that the relation on $x, y \in C$ "there is a chain between $x$ and $y$ " is symmetric and transitive.

Let $B$ consists of all $c \in C$ for which there exist chains between all elements of $A$ and $c$. Since $D$ contains all positive distances between elements of $A$, for distinct $a, b \in A, a, b$ is a chain as is $b, a$. Thus, the inclusion

$$
A \subseteq B
$$

follows. (We use here the fact that $A$ has at least 2 elements.) Moreover, it also follows that if there exists a chain between an element $c$ of $C$ and some element of $A$, then there exist chains between $c$ and all elements of $A$, that is, $c \in B$. We will deduce from it that

$$
\text { if } p \text { is a partial isometry of } A \text {, then } \tilde{p}(B)=B \text {. }
$$

Since we are dealing with finite sets, it will suffice to show $\tilde{p}(B) \subseteq B$. If $p$ is the partial isometry with empty domain, $\tilde{p}$ is the identity, and there is nothing to prove. Let $p$ be a partial automorphism of $A$ with non-empty domain. Pick a point $\bar{a}$ in the domain of $p$. Fix $b \in B$ in order to show that $\tilde{p}(b) \in B$. We can fix a chain $\bar{a}=c_{0}, c_{1}, \ldots, c_{n}=b$ is between $\bar{a}$ and $b$. Since $\tilde{p}$ is an automorphism of $C, \tilde{p}(\bar{a})=\tilde{p}\left(c_{0}\right), \tilde{p}\left(c_{1}\right), \ldots, \tilde{p}\left(c_{n}\right)=\tilde{p}(b)$ is a chain between $\tilde{p}(\bar{a})=p(\bar{a}) \in A$ and $\tilde{p}(b)$. Thus, there is a chain between $\tilde{p}(b)$ and some element of $A$, therefore, $\tilde{p}(b) \in B$.

Define a metric $\rho$ on $B$ by letting $\rho(a, b)$ be 0 if $a=b$ and otherwise be the infimum of the quantities

$$
r_{1}+\cdots+r_{n}
$$

where for some chain $c_{0}, c_{1}, \ldots, c_{n}$ between $a$ and $b$ we have

$$
C \models\left(R_{r_{i}}\left(c_{i-1}, c_{i}\right) \text { and } R_{r_{i}}\left(c_{i}, c_{i-1}\right)\right) \text { for all } 1 \leq i \leq n .
$$

By definition of $B, \rho$ is defined on all pairs $a, b$ of elements of $B$. Moreover, $\rho$ is clearly a metric. For each partial isometry $p$ of $A, \tilde{p} \uparrow B$ maps $B$ to $B$ by (2). In fact, $\tilde{p}\lceil B$ is an isometry of $B$ with $\rho$ since the image of a chain under $\tilde{p}$ and $\tilde{p}^{-1}$, which are automorphisms of $C$, is a chain. Of course, $\tilde{p} \uparrow B$ extends $p$. By (1) we have $A \subseteq B$. It remains to check that $\rho$ restricted to $A$ coincides with $d$, that is, $\rho(a, b)=d(a, b)$ for any distinct $a, b \in A$. The inequality $\leq$ is clear since $a, b$ is a chain. To see the other inequality, assume towards contradiction that $\rho(a, b)<d(a, b)$. This allows us to fix a chain $c_{0}, c_{1}, \ldots, c_{n} \in C$ between $a$ and $b$ and $r_{i} \in D$ for $1 \leq i \leq n$ so that

$$
r_{1}+\cdots+r_{n}<d(a, b)
$$


and (3) holds. Then the sequence $d(a, b), r_{1}, \ldots, r_{n}$ is a configuration. Let us call it $\alpha$. We then have a weak homomorphism from $M_{\alpha}$ to $C$ (whose range is $\left.\left\{c_{0}, \ldots, c_{n}\right\}\right)$ which contradicts the fact that $C$ is $\mathcal{T}$-free.

The moreover part of the theorem is clear from the definition of $\rho$.

\section{The CASE OF ONE PARTIAL ISOMETRY}

In this section, we deal with the situation when a single partial isometry $p$ on a finite metric space $A$ is given. We show in Theorem 3.2 how to embed $A$ into a finite metric space $B$ in such a way that $p$ extends to an isometry $\tilde{p}$ of $B$ so that for some $M \in \mathbb{N}, A$ and $\tilde{p}^{M}(A)$ are as "independent" from each other as possible. The problem of finding this type of extension in special case came up in the work of Kechris and Rosendal [5]. In fact, in Corollaries 4.1 and 4.4, we show how this extension theorem translates into properties of the isometry groups of the rational Urysohn metric space and the Urysohn metric space.

We recall a definition of an amalgam of two metric spaces. Let $A, B, C$ be finite metric spaces and let $f_{1}: A \rightarrow B$ and $f_{2}: A \rightarrow C$ be isometric embeddings and let $d_{1}$ and $d_{2}$ be the metrics on $B$ and $C$, respectively. We allow here $A$ to be empty in which case $f_{1}$ and $f_{2}$ are the empty functions. The amalgam of $B$ and $C$ over $\left(A, f_{1}, f_{2}\right)$ is defined as follows. Take the disjoint union of $B$ and $C$ and identify $f_{1}(a)$ with $f_{2}(a)$ for any $a \in A$. Call the quotient set $D$. There are natural injections $g_{1}: B \rightarrow D$ and $g_{2}: C \rightarrow D$. Define a metric $d$ on $D$ by transferring $d_{1}$ from $B$ to $g_{1}(B)$ by $g_{1}$ and $d_{2}$ from $C$ to $g_{2}(C)$ by $g_{2}$ and by defining $d\left(g_{1}(b), g_{2}(c)\right)$ for $b \in B$ and $c \in C$ as follows:

if $A \neq \emptyset, d\left(g_{1}(b), g_{2}(c)\right)=\min \left\{d_{1}\left(b, f_{1}(a)\right)+d_{2}\left(f_{2}(a), c\right): a \in A\right\}$;

if $A=\emptyset, d\left(g_{1}(b), g_{2}(c)\right)=\operatorname{diam}(B)+\operatorname{diam}(C)$.

We leave it to the reader to check that $d$ is well defined and that it is a metric.

The following lemma is the reason for importance of the amalgam. Its proof is straightforward and is left to the reader.

Lemma 3.1. Let a metric space $D$ be the amalgam of $B$ and $C$ over $\left(A, f_{1}, f_{2}\right)$. Let $g_{1}: B \rightarrow D$ and $g_{2}: C \rightarrow D$ be the natural isometric embeddings. Let $\phi: A \rightarrow A$ be an isometry and let $\psi_{1}, \psi_{2}$ be partial isometries of $B$ and $C$, respectively, extending $f_{1} \circ \phi \circ f_{1}^{-1}$ and $f_{2} \circ \phi \circ f_{2}^{-1}$, respectively. Then $g_{1} \circ \psi_{1} \circ g_{1}^{-1}$ and $g_{2} \circ \psi_{2} \circ g_{2}^{-1}$ have a common extension to a partial isometry of $D$.

Let $A$ be a finite metric space, let $D, E \subseteq A$, and let $p: D \rightarrow E$ be a partial isometry of $A$. We say that $x \in A$ is a cyclic point of $p$ if $p^{n}(x) \in D$ for each $n \in \mathbb{N}$. An $x \in A$ which is not cyclic is called acyclic. By $Z(p)$ we denote the set of all cyclic points of $p$.

Theorem 3.2. Let a finite metric space $A$ and a partial isometry $p$ of $A$ be given. There exist a finite metric space $B$ with $A \subseteq B$ as metric spaces, an isometry $\tilde{p}$ of $B$ extending $p$, and a natural number $M$ such that 
(i) $\tilde{p}^{2 M}=i d_{B}$;

(ii) if $a \in A$ is acyclic, then $\tilde{p}^{j}(a) \neq a$ for all $0<j<2 M$;

(iii) $A \cup \tilde{p}^{M}(A)$ is the amalgam of $A$ and $\tilde{p}^{M}(A)$ over $\left(Z(p), i d_{Z(p)}, \tilde{p}^{M} \uparrow\right.$ $Z(p))$.

Moreover, the distances in $B$ are in the additive semigroup generated by the distances in $A$.

Remark 3.1. Point (iii) above is a concise formulation of the following statement: $A \cap \tilde{p}^{M}(A)=Z(p), \tilde{p}^{M}\left\lceil Z(p)=\operatorname{id}_{Z(p)}\right.$, and if $d$ is the metric on $A$ and $\rho$ is the one on $B$, then, for $a_{1}, a_{2} \in A, \rho\left(a_{1}, \tilde{p}^{M}\left(a_{2}\right)\right)$ is equal to $2 \operatorname{diam}(A)$ or $\min \left\{d\left(a_{1}, z\right)+d\left(z, a_{2}\right): z \in Z(p)\right\}$ depending on whether $Z(p)$ is empty or not. Note that the last of these three conditions implies the first one.

Proof of Theorem 3.2. Let $\Delta=\operatorname{diam}(A)$, and let $\delta$ be the minimal value of $d(x, y)$ with $x \neq y, x, y \in A$. Put $Z=Z(p)$.

Let $D, E \subseteq A$ and let $p: D \rightarrow E$ be a partial isometry of $A$. Let $M$ be a natural number whose value will be chosen later. Consider $X=\{0, \ldots, 2 M-1\} \times A$. We start with defining an equivalence relation $\equiv$ on $X$. For $(x, m),(y, n) \in X$, let $(x, m) \equiv(y, n)$ if there exists $r \geq 0$ such that $p^{k}(x) \in D$ for all $0 \leq k<r$, $p^{r}(x)=y$, and $n+r=m \bmod 2 M$ or the same condition holds with the roles of $x$ and $y$ interchanged. It is easy to see that this is an equivalence relation. (Note that we can take $r=0$ so $(x, m) \equiv(x, m)$.) Set $B=X / \equiv$.

Define now a function $P: X \rightarrow X$ by letting

$$
P(x, n)=(x, n+1 \bmod 2 M) .
$$

It is easy to see that $P$ respects the relation $\equiv$. Let $\tilde{p}$ be the function induced by $P$ on $B=X / \equiv$.

We describe now a metric $\rho$ on $B$. If $[x, m]$ and $[y, n]$ are two equivalence classes of $\equiv$, define $\rho([x, m],[y, n])$ to be the minimum of $2 \Delta$ and the sums of the following sort

$$
\sum_{i=0}^{k-1} d\left(x_{i}, y_{i+1}\right)
$$

where $x_{0}=x, x_{k}=y, n_{0}=m, n_{k}=n$, and for some numbers $0 \leq n_{i}<2 M$, for $0 \leq i \leq k,\left(y_{i}, n_{i-1}\right) \equiv\left(x_{i}, n_{i}\right)$ for all $1 \leq i \leq k$. Note that the sum in (4) is equal to 0 precisely when $x_{i}=y_{i+1}$ for all $0 \leq i \leq n-1$ which implies that $(x, m) \equiv(y, n)$. This shows that the function $\rho$ is well defined on $B$ (it does not depend on the choice of the representatives $(x, m)$ and $(y, n))$ and that $\rho([x, m],[y, n])=0$ implies $[x, m]=[y, n]$. It is straightforward to check that $[x, m]=[y, n]$ implies $\rho([x, m],[y, n])=0$, that $\rho$ is symmetric and that it fulfills the triangle inequality. Thus, $\rho$ is a metric on $B$. It is also easy to see that $\tilde{p}$ is 
an isometry. Notice also that the distances with respect to $\rho$ are in the additive semigroup of the distances with respect to $d$.

We define a function $h: A \rightarrow B$ by $h(x)=[x, 0]$. We will prove below that, with an appropriate choice of $M, h$ is an isometry. In particular, it is one-to-one and it is easy to see that if we identify $A$ with $h(A) \subseteq B$, then $\tilde{p}$ extends $p$. Point (i) is immediate from the definition of $P$. To see (ii), fix $0 \leq j<2 M$ and assume that $\tilde{p}^{j}([x, 0])=[x, 0]$. This implies that $(x, j) \equiv(x, 0)$ so

$$
p^{j}(x)=x \text { or } p^{2 M-j}(x)=x .
$$

In the first case, we of course have $p^{i}(x) \in D$ for all $0 \leq i<j$ and in the second case $p^{i}(x) \in D$ for all $0 \leq i<2 M-j$. Since $x$ is acyclic, it follows that $j=0$ or $2 M-j=0$, which proves (ii).

It remains to show that $h$ is an isometry and that (iii) holds. Both these arguments require computations with the metrics $\rho$ and $d$ which will be done in Claims 1 and 2 below. The following notion will be useful. For $0 \leq m<2 M$, we call a sequence $x_{0}, x_{1}, \ldots x_{k} \in A$ together with $r_{1}, \ldots, r_{k} \in \mathbb{Z}$ an $m$-chain between $x$ and $y$ if $x_{0}=x, x_{k}=y, \sum_{i=1}^{k} r_{i}=m \bmod 2 M$ and, if $r_{i}>0$, then $x_{i}, p\left(x_{i}\right), \ldots, p^{r_{i}-1}\left(x_{i}\right) \in D$, and, if $r_{i}<0$, then $x_{i}, p^{-1}\left(x_{i}\right), \ldots, p^{r_{i}+1}\left(x_{i}\right) \in E$. It is easy to see that the definition of $\rho([x, 0],[y, m])$ can be reformulated as

$$
\min \left(2 \Delta, \min \sum_{i=0}^{k-1} d\left(x_{i}, p^{r_{i+1}}\left(x_{i+1}\right)\right)\right)
$$

where the second minimum is taken over all $m$-chains between $x$ and $y$.

Let $x_{0}, x_{1}, \ldots, x_{k}, r_{1}, \ldots, r_{k}$ and $y_{0}, y_{1}, \ldots, y_{l}, q_{1}, \ldots, q_{l}$ be two $m$-chains between $x$ and $y$. Define the second one to be shorter than the first one if

$$
\sum_{i=0}^{l-1} d\left(y_{i}, p^{q_{i+1}}\left(y_{i+1}\right)\right) \leq \sum_{i=0}^{k-1} d\left(x_{i}, p^{r_{i+1}}\left(x_{i+1}\right)\right)
$$

and either $l<k$ or $l=k, q_{l}=0$ and $r_{k} \neq 0$.

Now we can state the first claim. In order to prove it, however, we need to specify the value of $M$. If $x \in A$ is cyclic, let $m_{x}$ be smallest $n>0$ with $p^{n}(x)=$ $x$. If $x \in A$ is acyclic, let $n_{x}=\min \left\{n \geq 0: p^{n}(x) \notin D\right\}+\max \left\{n: p^{-n}(x) \in D\right\}$. Let $M$ be a positive natural number divisible by all the $m_{x}$ 's for cyclic $x \in A$ and such that

$$
\left(M-\max \left\{n_{x}: x \in A, x \text { acyclic }\right\}\right) \delta>\left(\max \left\{n_{x}: x \in A, x \text { acyclic }\right\}\right) 2 \Delta
$$

with the convention that $\max \emptyset=0$.

Claim 1. Let $x_{0}, x_{1}, \ldots, x_{k}, r_{1}, \ldots, r_{k}$ be an $m$-chain between $x$ and $y$ which cannot be made shorter. Then the following three conditions hold:

(a) if some $x_{i}$ with $1 \leq i \leq k$ is cyclic, then $x_{1}$ is cyclic and ( $k=1$ or $(k=2$ and $\left.r_{2}=0\right)$ ); 
(b) $p^{r_{i+1}}\left(x_{i+1}\right) \neq x_{i}$ for all $1 \leq i \leq k-1$;

(c) $r_{i} \geq 0$ for all $1 \leq i \leq k$ or $r_{i} \leq 0$ for all $1 \leq i \leq k$.

Proof of Claim 1. (a) Let $x_{i}$ be cyclic and $i \geq 1$. We assume that the conclusion of the implication stated in (a) fails and show how to define an $m$ chain between $x$ and $y$ which is shorter than $x_{0}, x_{1}, \ldots, x_{k}, r_{1}, \ldots, r_{k}$. Define the new $m$-chain as follows:

if $2 \leq i$,

$$
\begin{aligned}
& y_{0}=x_{0}, \ldots, y_{i-2}=x_{i-2}, y_{i-1}=x_{i}, y_{i}=x_{i+1}, \ldots, y_{k-1}=x_{k}, \\
& q_{1}=r_{1}, \ldots, q_{i-2}=r_{i-2}, q_{i-1}=r_{i-1}+r_{i}, q_{i}=r_{i+1}, \ldots, q_{k-1}=r_{k},
\end{aligned}
$$

if $k=i+1$ and $r_{k} \neq 0$,

$$
\begin{aligned}
& y_{0}=x_{0}, \ldots, y_{i-1}=x_{i-1}, y_{i}=p^{-r_{i+1}}\left(x_{i}\right), y_{k}=x_{k}, \\
& q_{1}=r_{1}, \ldots, q_{i-1}=r_{i-1}, q_{i}=r_{i}+r_{i+1}, q_{k}=0,
\end{aligned}
$$

and if $k-2 \geq i$,

$$
\begin{aligned}
& y_{0}=x_{0}, \ldots, y_{i-1}=x_{i-1}, y_{i}=p^{-r_{i+1}}\left(x_{i}\right), y_{i+1}=x_{i+2}, \ldots, y_{k-1}=x_{k}, \\
& q_{1}=r_{1}, \ldots, q_{i-1}=r_{i-1}, q_{i}=r_{i}+r_{i+1}, q_{i+1}=r_{i+2}, \ldots, q_{k-1}=r_{k} .
\end{aligned}
$$

Note that since $x_{i}$ is cyclic, $p^{j}\left(x_{i}\right)$ is defined for each $j \in \mathbb{Z}$, so the definitions above make sense. Clearly they define $m$-chains from $x$ to $y$. It is obvious from their definitions that to show that the new $m$-chain is shorter than the old one, it suffices to check relation (6). In the first case $(2 \leq i)$, this amounts to the following calculation (with the last equality following from $p$ being an isometry)

$$
\begin{aligned}
d\left(y_{i-2}, p^{q_{i-1}}\left(y_{i-1}\right)\right) & =d\left(x_{i-2}, p^{r_{i-1}+r_{i}}\left(x_{i}\right)\right) \\
& \leq d\left(x_{i-2}, p^{r_{i-1}}\left(x_{i-1}\right)\right)+d\left(p^{r_{i-1}}\left(x_{i-1}\right), p^{r_{i-1}+r_{i}}\left(x_{i}\right)\right) \\
& =d\left(x_{i-2}, p^{r_{i-1}}\left(x_{i-1}\right)\right)+d\left(x_{i-1}, p^{r_{i}}\left(x_{i}\right)\right) .
\end{aligned}
$$

In the other two cases, we have

$$
d\left(y_{i-1}, p^{q_{i}}\left(y_{i}\right)\right)=d\left(x_{i-1}, p^{r_{i}}\left(x_{i}\right)\right) .
$$

Moreover, if $k=i+1$ and $r_{k} \neq 0$,

$$
d\left(y_{i}, p^{0}\left(y_{k}\right)\right)=d\left(p^{-r_{i+1}}\left(x_{i}\right), x_{i+1}\right)=d\left(x_{i}, p^{r_{i+1}}\left(x_{i+1}\right)\right)
$$

where the last equality follows from $p$ being an isometry. If $i \leq k-2$, we have

$$
\begin{aligned}
d\left(y_{i}, p^{q_{i+1}}\left(y_{i+1}\right)\right) & =d\left(p^{-r_{i+1}}\left(x_{i}\right), p^{r_{i+2}}\left(x_{i+2}\right)\right) \\
& \leq d\left(p^{-r_{i+1}}\left(x_{i}\right), x_{i+1}\right)+d\left(x_{i+1}, p^{r_{i+2}}\left(x_{i+2}\right)\right) \\
& =d\left(x_{i}, p^{r_{i+1}}\left(x_{i+1}\right)\right)+d\left(x_{i+1}, p^{r_{i+2}}\left(x_{i+2}\right)\right)
\end{aligned}
$$

the last equality following from the fact that $p$ is an isometry. Thus, in either of these two cases $\left(k=i+1\right.$ and $r_{k} \neq 0$ or $\left.i \leq k-2\right)$ we see that (6) holds. 
(b) This point is obvious. If the condition fails for some $1 \leq i \leq k-1$, to construct an $m$-chain between $x$ and $y$ shorter than $x_{0}, x_{1}, \ldots, x_{k}, r_{1}, \ldots, r_{k}$ simply take $y_{j}=x_{j}$ if $j \leq i-1, y_{j}=x_{j+1}$ if $j>i-1, q_{j}=r_{j}$ if $j \leq i-1$, $q_{i}=r_{i}+r_{i+1}$, and $q_{j}=r_{j+1}$ if $k-1 \geq j>i$.

(c) It is obvious that $r_{i} \neq 0$ for all $1 \leq i \leq k-1$ since otherwise $y_{j}=x_{j}$, $r_{j}=p_{j}$ for $j<i$ and $y_{j}=x_{j+1}, r_{j}=p_{j+1}$ for $i \leq j \leq k-1$ would define an $m$-chain between $x$ and $y$ shorter than $x_{0}, x_{1}, \ldots, x_{n}, r_{1}, \ldots, r_{n}$. Now assume towards contradiction that $r_{i}>0$ and $r_{i+1}<0$ for some $1 \leq i \leq k-1$. (The other case is dealt with in a similar manner.) If $r_{i} \leq\left|r_{i+1}\right|$, define an $m$-chain between $x$ and $y$ by letting

$$
\begin{array}{r}
y_{0}=x_{0}, \ldots, y_{i-1}=x_{i-1}, y_{i}=x_{i+1}, \ldots, y_{k-1}=x_{k} \\
q_{1}=r_{1}, \ldots, q_{i-1}=r_{i-1}, q_{i}=r_{i}+r_{i+1}, \ldots, q_{k-1}=r_{k} .
\end{array}
$$

Then we have

$$
d\left(y_{i-1}, p^{q_{i}}\left(y_{i}\right)\right)=d\left(x_{i-1}, p^{r_{i}+r_{i+1}}\left(x_{i+1}\right)\right) \leq d\left(x_{i-1}, p^{r_{i}}\left(x_{i}\right)\right)+d\left(x_{i}, p^{r_{i+1}}\left(x_{i+1}\right)\right)
$$

which justifies (6) showing that the new $m$-chain is shorter than $x_{0}, x_{1}, \ldots, x_{n}$, $r_{1}, \ldots, r_{n}$. Note that $p^{r_{i}+r_{i+1}}\left(x_{i+1}\right)$ makes sense since $0 \geq r_{i}+r_{i+1} \geq r_{i+1}$.

If $r_{i} \geq\left|r_{i+1}\right|>0$, define the chains in the case $k=i+1$ and in the case $k-2 \geq i$ exactly as in point (a) in the analogous cases. Note that the definition $y_{i}=p^{-r_{i+1}}\left(x_{i}\right)$ makes sense since $0 \leq-r_{i+1} \leq r_{i}$ and it is assumed that $p^{j}\left(x_{i}\right) \in D$ for all $0 \leq j<r_{i}$. It is easy to see that what is defined here are $m$-chains between $x$ and $y$. The calculations from point (a) show that the new $m$-chains are shorter than the old ones. The claim follows.

We say that the distance $\rho([x, 0],[y, m])$ is realized on an $m$-chain $x_{0}, \ldots, x_{k}$, $r_{1}, \ldots, r_{k}$ between $x$ and $y$ if

$$
\rho([x, 0],[y, m])=\sum_{i=0}^{k-1} d\left(x_{i}, p^{r_{i+1}}\left(x_{i+1}\right)\right) .
$$

In particular, the second minimum in (5) is less than or equal to $2 \Delta$.

Claim 2. Assume that the distance $\rho([x, 0],[y, m])$ is realized on an $m$-chain $x_{0}, \ldots, x_{k}, r_{1}, \ldots, r_{k}$ between $x$ and $y$. Then one of the following two conditions holds:

(a) $x_{1}$ is cyclic and $\left(k=1\right.$ or $\left(k=2\right.$ and $\left.\left.r_{2}=0\right)\right)$;

(b) $\sum_{i=1}^{n}\left|r_{i}\right|<M$.

Proof of Claim 2. It is clear that the relation between $m$-chains of being shorter does not have cycles, therefore if a distance is realized on a chain, it is realized on a chain which cannot be made shorter. Thus, the $m$-chain $x_{0}, x_{1}, \ldots, x_{k}, r_{1}, \ldots, r_{k}$ between $x$ and $y$ cannot be shortened. Therefore, by 
Claim 1, it suffices to show that the assumption that all $x_{i}$ with $1 \leq i \leq k-1$ are acyclic and conditions Claim 1(b),(c) imply Claim 2(b). Notice that

$$
k \max \left\{n_{x}: x \in A\right\}<M
$$

since otherwise by Claim 1(b) and (7)

$$
\begin{aligned}
\rho([x, 0],[y, m]) & =\sum_{i=0}^{k-1} d\left(x_{i}, p^{r_{i+1}}\left(x_{i+1}\right)\right) \geq(k-1) \delta \\
& \geq\left(\frac{M}{\max \left\{n_{x}: x \text { acyclic }\right\}}-1\right) \delta>2 \Delta
\end{aligned}
$$

contradicting $\rho([x, 0],[y, m]) \leq 2 \Delta$. By Claim $1(\mathrm{c})$, we can assume $r_{i} \geq 0$ for all $1 \leq i \leq k$. (The case $r_{i} \leq 0$ for all $1 \leq i \leq k$ is handled similarly.) Each $x_{i}$ acyclic and therefore $0 \leq r_{i} \leq n_{x_{i}}$ whence by (8)

$$
\sum_{i=1}^{k} r_{i} \leq k \max \left\{n_{x}: x \text { acyclic }\right\}<M
$$

and Claim 2 follows.

We check now that $h$ is an isometry which amounts to proving that

$$
\rho([x, 0],[y, 0])=d(x, y) .
$$

Note that since $x_{0}=x, x_{1}=y$ and $r_{1}=0$ is a 0 -chain between $x$ and $y$,

$$
\rho([x, 0],[y, 0]) \leq d(x, y)<2 \Delta
$$

holds obviously and implies that $\rho([x, 0],[y, 0])$ is realized on a 0 -chain. Fix such a 0 -chain $x_{0}, x_{1}, \ldots, x_{k}, r_{1}, \ldots, r_{k}$ between $x$ and $y$.

If Claim 2(a) holds for this chain, then we have $r_{1}=\sum_{i=1}^{k} r_{i}=0 \bmod 2 M$, hence $m_{x_{1}} \mid r_{1}$ and therefore $p^{r_{1}}\left(x_{1}\right)=x_{1}$. If $k=1$, we get $d\left(x_{0}, p^{r_{1}}\left(x_{1}\right)\right)=$ $d\left(x_{0}, x_{1}\right)=d(x, y)$. If $k=2$, since $r_{2}=0$, we have

$$
d\left(x_{0}, p^{r_{1}}\left(x_{1}\right)\right)+d\left(x_{1}, p^{r_{2}}\left(x_{2}\right)\right)=d\left(x_{0}, x_{1}\right)+d\left(x_{1}, x_{2}\right) \geq d(x, y) .
$$

Thus, $d(x, y) \leq \rho([x, 0],[y, 0])$.

If Claim 2(b) holds, then, since $\sum_{i=1}^{k} r_{i}=0 \bmod 2 M$, we get $r_{i}=0$ for each $1 \leq i \leq k$. Thus,

$$
\sum_{i=0}^{k-1} d\left(x_{i}, p^{r_{i+1}}\left(x_{i+1}\right)\right)=\sum_{i=0}^{k-1} d\left(x_{i}, x_{i+1}\right) \geq d\left(x_{0}, x_{k}\right)=d(x, y),
$$

and we are done.

To check point (iii), we need to prove that $\tilde{p}^{M} \uparrow Z=\mathrm{id}_{Z}$ and to compute $\rho([x, 0],[y, M])$. (See the remark following the statement of Theorem 3.2.) For $x \in Z, \tilde{p}^{M}(x)=p^{M}(x)=x$ since $m_{x}$ divides $M$.

Now we compute $\rho([x, 0],[y, M])$. If this distance were realized on an $M$-chain $x_{0}, x_{1}, \ldots, x_{k}, r_{1}, \ldots, r_{k}$ between $x$ and $y$ fulfilling Claim $2(\mathrm{~b})$, then $\sum_{i=1}^{n} r_{i}=$ 
$M \bmod 2 M$ leads to a contradiction. Thus, if the distance is realized on an $M$-chain, the chain fulfills Claim 2(a). We have now two situations: either $Z$ is empty or not.

Assume first that $Z=\emptyset$. Since there are no cyclic points, $\rho([x, 0],[y, M])$ is not realized on an $M$-chain fulfilling Claim 2(a). Thus, it is equal to $2 \Delta$.

Assume now $Z \neq \emptyset$. If an $M$-chain $x_{0}, x_{1}, \ldots, x_{k}, r_{1}, \ldots, r_{k}$ realizes the distance $\rho([x, 0],[y, M])$, then $x_{1}$ is cyclic and either $k=1$ or $k=2$ and $r_{2}=0$. In either case $r_{1}=M \bmod 2 M$ whence $M$ divides $r_{1}$ and so $m_{x_{1}}$ divides $r_{1}$. Therefore, $p^{r_{1}}\left(x_{1}\right)=x_{1}$. If $k=1, y$ is cyclic and

$$
\rho([x, 0],[y, M])=d\left(x_{0}, x_{1}\right)=d(x, y) .
$$

If $k=2$, it follows that

$$
\begin{aligned}
\rho([x, 0],[y, M]) & =\min \left(2 \Delta, \min \left\{d\left(x, x_{1}\right)+d\left(x_{1}, y\right): x_{1} \in Z\right\}\right) \\
& =\min \left\{d\left(x, x_{1}\right)+d\left(x_{1}, y\right): x_{1} \in Z\right\}
\end{aligned}
$$

as required.

Remark 3.2. The definition of the set underlying the metric space $B$ and of $\tilde{p}$ draws on ideas which are already present in Mackey's construction of induced action [6, p.190] (see also [1, 2.3.5]). In the context of extensions of partial isomorphisms of finite graphs, a similar definition was used by Hrushovski in [3]. The new ingredients here are the choice of $M$, the definition of the metric on $B$ and the arguments concerning it.

\section{Consequences for isometry Groups}

The present section contains derivations from Theorems 2.1 and 3.2 of properties of the structure of conjugacy classes of the isometry groups of the rational Urysohn metric space and the Urysohn metric space (Corollaries 4.1 and 4.4). These properties have broader consequences as described in Corollaries 4.3 and 4.5 .

Let $G$ be a Polish group and let $n \in \mathbb{N}$. By the diagonal action of $G$ on $G^{n}$ we understand the action

$$
G \times G^{n} \ni\left(g,\left(h_{1}, \ldots, h_{n}\right)\right) \rightarrow\left(g h_{1} g^{-1}, \ldots, g h_{n} g^{-1}\right) \in G^{n} .
$$

This is a generalization of the conjugacy action of $G$ on itself which we obtain by setting $n=1$ in the above definition. Slightly abusing the notation, we will write $g \bar{h} f$ for $\left(g h_{1} f, \ldots, g h_{n} f\right)$ where $g, f \in G$ and $\bar{h}=\left(h_{1}, \ldots, h_{n}\right) \in G^{n}$. We say that $\bar{h} \in G^{n}$ is cyclically dense for the diagonal action of $G$ on $G^{n}$ if for some $g \in G,\left\{g^{k} \bar{h} g^{-k}: k \in \mathbb{N}\right\}$ is dense in $G^{n}$. A point $\bar{h} \in G^{n}$ is generic for the diagonal action of $G$ on $G^{n}$ if its orbit with respect to this action is a dense $G_{\delta}$. 
If $X$ is a Polish metric space, by $\operatorname{Iso}(X)$ we denote the group of all isometries of $X$ with the pointwise convergence topology. With this topology $\operatorname{Iso}(X)$ is a Polish group with composition as group operation. If $A \subseteq X$, by $\operatorname{Iso}_{A}(X)$ we denote the closed subgroup of $\operatorname{Iso}(X)$ consisting of all the elements which fix each point in $A$.

Corollary 4.1. Let $A \subseteq \mathbb{U}_{0}$ be finite. For each $n \in \mathbb{N}$ the diagonal action of $I_{\text {Iso }}\left(\mathbb{U}_{0}\right)$ on Iso ${ }_{A}\left(\mathbb{U}_{0}\right)^{n}$ has a point which is cyclically dense and generic.

We start with a lemma.

Lemma 4.2. Let $A \subseteq \mathbb{U}_{0}$ be finite and let $n \in \mathbb{N}$. Assume that $\bar{\phi} \in \operatorname{Iso}(A)^{n}$ and $\chi \in I s o(A)$ are given. Then there exist $\bar{f} \in I$ Iso $\left(\mathbb{U}_{0}\right)^{n}$ and $h \in I s o\left(\mathbb{U}_{0}\right)$ extending $\bar{\phi}$ and $\chi$, respectively, such that

$$
\overline{\left\{h^{m} \bar{f} h^{-m}: m \in \mathbb{N}\right\}}=\left\{\bar{g} \in I s o\left(\mathbb{U}_{0}\right)^{n}: \exists m \in \mathbb{N} \bar{g}\left\lceil A^{n}=\chi^{m} \bar{\phi} \chi^{-m}\right\} .\right.
$$

Proof. The corollary is derived from Theorems 2.1 and 3.2 by a back-and-forth argument. An isometry between two finite subsets of a $\mathbb{U}_{0}$ will be called a finite isometry. Recall that for a finite isometry $p, Z(p)$ stands for the set of all cyclic points of $p$, that is, points $x$ for which $p^{i}(x)$ is defined for all $i \in \mathbb{N}$.

The inclusion $\subseteq$ holds for any isometric extensions of $\bar{\phi}$ and $\chi$. Therefore, we construct such extensions $\bar{f}$ and $h$ so that the opposite inclusion holds. In fact, it suffices to make sure that for any $n$-tuple of finite isometries $\bar{\gamma}$ extending $\bar{\phi}$ there is $m \in \mathbb{N}$ with $h^{m} \bar{f} h^{-m}$ extending $\bar{\gamma}$. By the standard back-and-forth inductive argument, we construct $\bar{f}$ and $h$ by producing $n$-tuples of finite isometries $\bar{\phi}_{k}$ and finite isometries $\chi_{k}, k \in \mathbb{N}$, such that $\bar{f}$ is the common extension of all the $\bar{\phi}_{k} \mathrm{~s}$ and $h$ is the common extension of all the $\chi_{k} \mathrm{~s}$. The inductive step, which produces $\phi_{k}$ and $\chi_{k}$ or $\bar{\phi}_{k}^{-1}$ and $\chi_{k}^{-1}$ depending on whether $k$ is even or odd, is equivalent to the following:

Assume we are given $x \in \mathbb{U}_{0}$ and a finite isometry $\chi^{\prime}$ with $Z\left(\chi^{\prime}\right)=A$. Assume that $\bar{\phi}^{\prime}$ and $\bar{\gamma}$ are $n$-tuples of finite isometries and that both extend $\bar{\phi}$. Then we can find a finite isometry $\chi^{\prime \prime}$ extending $\chi^{\prime}$ and an $n$-tuple of finite isometries $\bar{\phi}^{\prime \prime}$ extending $\bar{\phi}^{\prime}$ such that the domains of $\chi^{\prime \prime}$ and of each component of $\bar{\phi}^{\prime \prime}$ contain $x, Z\left(\chi^{\prime \prime}\right)=A$ and, for some $M \in \mathbb{N}, \bar{\phi}^{\prime \prime}\left(\chi^{\prime \prime}\right)^{-M}$ extends $\left(\chi^{\prime \prime}\right)^{-M} \bar{\gamma}$.

We accomplish it as follows. Let $A_{1}$ be the union of $\{x\}$ and the domains and ranges of $\chi^{\prime}$ and of the components of the $n$-tuples $\bar{\gamma}$ and $\bar{\phi}^{\prime}$. Use Theorem 2.1 to find an extension of $\bar{\phi}^{\prime}$ to $\bar{\phi}_{1}^{\prime \prime} \in \operatorname{Iso}\left(A_{2}\right)^{n}$ with $A_{1} \subseteq A_{2}$ and with $A_{2}$ finite. (Note that by Theorem 2.1 we can find a finite rational metric space $A_{2}$ isometrically embedding $A_{1}$ as above. By universality and ultrahomegeneity of $\mathbb{U}_{0}$ with respect to finite rational metric spaces, we can assume that $A_{1} \subseteq A_{2} \subseteq \mathbb{U}_{0}$.) Now use Theorem 3.2 to find a natural number $M$ and a finite isometric extension $\chi_{1}^{\prime \prime}: B \rightarrow B$ of $\chi^{\prime}$ with $A_{2} \subseteq B$. (Again universality and ultrahomogeneity of 
$\mathbb{U}_{0}$ are used here.) Define

$$
\chi^{\prime \prime}=\chi_{1}^{\prime \prime} \uparrow \bigcup_{0 \leq i \leq M-1}\left(\chi_{1}^{\prime \prime}\right)^{i}\left(A_{2}\right) .
$$

The function $\bar{\phi}_{1}^{\prime \prime}$ is defined on $A_{1}^{n}$ while $\left(\chi_{1}^{\prime \prime}\right)^{-M} \bar{\gamma}\left(\chi_{1}^{\prime \prime}\right)^{M}$ is defined on $\left(\chi_{1}^{\prime \prime}\left(A_{1}\right)\right)^{n}$ (by Theorem 3.2(i)). In particular, both these functions contain $Z\left(\chi^{\prime}\right)^{n}=A^{n}$ in their domains. The restriction of $\bar{\phi}_{1}^{\prime \prime}$ to $A^{n}$ is equal to $\bar{\phi}$ as is, by Theorem 3.2(iii), the restriction $\left(\chi_{1}^{\prime \prime}\right)^{-M} \bar{\gamma}\left(\chi_{1}^{\prime \prime}\right)^{M}$ to $Z\left(\chi^{\prime}\right)^{n}=A^{n}$. Let $\bar{\phi}^{\prime \prime}$ be an $n$ tuple of finite isometries which is a common extension of $\bar{\phi}_{1}^{\prime \prime}$ and $\left(\chi_{1}^{\prime \prime}\right)^{-M} \bar{\gamma}\left(\chi_{1}^{\prime \prime}\right)^{M}$ and which exists by Theorem 3.2(iii) and Lemma 3.1. Also by Theorem 3.2(ii), we have $Z\left(\chi^{\prime \prime}\right)=Z\left(\chi^{\prime}\right)$. Now $\bar{\phi}^{\prime \prime}$ and $\chi^{\prime \prime}$ are as required.

Proof of Corollary 4.1. Fix $n$ and consider the diagonal action of $\operatorname{Iso}_{A}\left(\mathbb{U}_{0}\right)$ on $\operatorname{Iso}_{A}\left(\mathbb{U}_{0}\right)^{n}$. It is easy to see that cyclically dense elements, once they exist, form a dense $G_{\delta}$. Thus, it suffices to prove the existence of a cyclically dense element and, separately, the existence of a generic element.

The existence of a cyclically dense element is just a special case of Lemma 4.2 with $\bar{\phi}$ being the $n$-tuple of the identity maps on $A$ and $\chi$ being the identity map on $A$.

Theorem 2.1 implies the existence of a generic element by the methods of [ 5 , Theorem 5.2]. A proof of this implication with $A=\emptyset$ is included in the remarks following Theorem 5.2 in [5]. The following simple argument derives the case of arbitrary finite $A$ from the particular case $A=\emptyset$. Let $C$ be a comeager orbit in Iso $\left(\mathbb{U}_{0}\right)^{n}$. Pick $\bar{h} \in C \cap \operatorname{Iso}_{A}\left(\mathbb{U}_{0}\right)^{n}$. Then for some $g_{0} \in \operatorname{Iso}\left(\mathbb{U}_{0}\right)$

$$
\left\{g g_{0} \bar{h} g_{0}^{-1} g^{-1}: g \in \operatorname{Iso}_{A}\left(\mathbb{U}_{0}\right)^{n}\right\}
$$

is contained in $\operatorname{Iso}_{A}\left(\mathbb{U}_{0}\right)^{n}$ and non-meager in it. It follows that $\bar{h}_{0}=g_{0} \bar{h} g_{0}^{-1}$ is in $\operatorname{Iso}_{A}\left(\mathbb{U}_{0}\right)^{n}$ and its orbit with respect to the diagonal action of $\operatorname{Iso}_{A}\left(\mathbb{U}_{0}\right)$ is non-meager. Since by (i) this action has a dense orbit, each non-meager orbit is in fact comeager, hence a dense $G_{\delta}$ by Effros' theorem (see [1, 2.2.2]).

The authors of [5] define a Polish group $G$ to have ample generics if for each $n \in \mathbb{N}$ the diagonal action of $G$ on $G^{n}$ has a generic element. So, Corollary 4.1 implies that Iso $\left(\mathbb{U}_{0}\right)$ has ample generics. As shown in [5, Section 5] existence of ample generics in a Polish group has strong consequences for the structure of the group. The following corollary is an immediate consequence of Corollary 4.1 and $[5$, Theorems 5.7, 5.9, 5.21].

Corollary 4.3. The Polish group Iso $\left(\mathbb{U}_{0}\right)$ has the following properties:

(i) any subgroup of it of index $<2^{\aleph_{0}}$ is open;

(ii) it is not the union of countably many cosets of non-open subgroups, in particular, it is not the union of a countable sequence of non-open subgroups; 
(iii) any homomorphism from it to a topological separable group is continuous.

Let $\mathbb{U}$ be the Urysohn metric space. Since by [4, Lemmas $6.19,6.20]$ the isometry group of $\mathbb{U}_{0}$ embeds into the isometry group of $\mathbb{U}$ as a dense subgroup, Corollary 4.1 implies the following result.

Corollary 4.4. All the diagonal actions of Iso(U) have cyclically dense elements.

Remark 4.1. The proof from [4] and the proof of Corollary 4.1 yield also that, given a finite subset $A$ of the Urysohn metric space $\mathbb{U}$, the diagonal actions of the group $\operatorname{Iso}_{A}(\mathbb{U})$ have cyclically dense elements. For other Polish groups with this property see [5, Theorem 1.10] and the references quoted in that paper.

Remark 4.2. Kechris and Rosendal [5, Theorem 1.2] and, independently, Glasner and Pestov proved a precursor to Corollaries 4.1 and 4.4. They showed that Iso $\left(\mathbb{U}_{0}\right)$ and Iso(U) have elements with dense conjugacy classes.

An interesting consequence of Corollary 4.4 which strengthens, for metric groups, a theorem of Morris and Pestov [8] was pointed out to me by Alekos Kechris. By [11] Iso(U) is a universal Polish group, that is, each Polish group is isomorphic to a closed subgroup of it. Now Corollary 4.4 for the diagonal action with $n=1$ implies immediately that $\operatorname{Iso}(\mathbb{U})$ is topologically 2-generated, that is, there are two elements of it which generate a dense subgroup. Thus, we obtain Corollary 4.5 below. This result implies that each metric separable group is contained in a metric separable topologically 2-generated group. This is a topological analog of the classical Higman-Neumann-Neumann theorem and was proved by a very different method by Morris and Pestov [8, Corollary $1]$.

Corollary 4.5. There exists a universal Polish group which is topologically 2-generated.

\section{REFERENCES}

[1] H. Becker, A.S. Kechris, The Descriptive Set Theory of Polish Group Actions, London Mathematical Society Lecture Note Series, 232, Cambridge University Press, 1996.

[2] B. Herwig, D. Lascar, Extending partial automorphisms and the profinite topology on free groups, Trans. Amer. Math. Soc. 352(1999), 1985-2021.

[3] E. Hrushovski, Extending partial isomorphisms of graphs, Combinatorica 12(1992), 411416.

[4] A.S. Kechris, V. Pestov, S. Todorcevic, Fraisse limits, Ramsey theory, and topological dynamics of automorphism groups, Geom. Funct. Anal., to appear.

[5] A.S. Kechris, C. Rosendal, Turbulence, amalgamation and generic automorphisms of homogeneous structures, to appear.

[6] G.W. Mackey, Ergodic theory and virtual groups, Math. Ann. 166(1966), 187-207. 
[7] D. Marker, Model Theory: An Introduction, Graduate Texts in Mathematics, Springer, 2002.

[8] S.A. Morris, V. Pestov, A topological generalization of the Higman-Neumann-Neumann theorem, J. Group Theory 1(1998), 181-187.

[9] V. Pestov, Ramsey-Milman phenomenon, Urysohn metric spaces, and extremely amenable groups, Israel J. Math. 127(2002), 317-357; A corrigendum to the article: Ramsey-Milman phenomenon, Urysohn metric spaces, and extremely amenable groups, Israel J. Math., to appear.

[10] P. Urysohn, Sur en espace métrique universel, Bull. Sci. Math. 51(1927), 43-64, 74-90.

[11] V.V. Uspenskij, On the group of isometries of the Urysohn universal metric space, Comment. Math. Univ. Carolin. 31(1990), 181-182.

Department of Mathematics, University of Illinois, 1409 W. Green St., Urbana, IL 61801

E-mail address: ssolecki@math.uiuc.edu 